Neonatal Audit Programme (NNAP) suggests use varies according to level of care but did not differentiate dependent on those transferred in.

Method IUT requests are coordinated by the regional transport team. Information was retrospectively reviewed from 01/11/16 to $31 / 12 / 16$, just after starting collection on magnesium sulphate use. Comparison was made from 01/03/17 to 30/04/17. Gestation, base hospital, risk of preterm delivery and maternal history was also recorded.

Results 189 cases were reviewed, 104 in 2016 and 85 in 2017. 35 were ineligible since gestation was $>34^{+0} / 40$. $9.2 \%$ patients had no information recorded.

In 2016, 8.5\% referrals received magnesium sulphate (5/59). This increased to $11.7 \%$ in 2017 (8/68). Only one patient was $>30^{+0} / 40$. Twenty neonatal units featured, two from outof-region. The greatest use was at a level 2 unit (43\%). 50\% units recorded no use. Most referrals followed rupture of membranes associated with contractions.

Conclusion Magnesium sulphate has clear benefit in the preterm population but appears to be under-utilised in our region. The majority of women met the criteria for magnesium but had not received it. Findings amongst the IUT group are below those reported by NNAP. Condition was only sought at the time of referral.

Recommendation Increased recognition of national guidance is required alongside inter-collaborative working with obstetric teams. Results have been disseminated at the network meeting and a guideline is in progress. The transport service may offer a unique opportunity to prompt use in this potentially vulnerable group.

\section{G118(P) ASSOCIATIONS BETWEEN POSTNATAL GROWTH RATES AND COGNITIVE OUTCOMES AT AGE 16 YEARS IN INFANTS BORN SMALL-FOR-GESTATIONAL-AGE AT TERM}

E Hallinan, M Fewtrell, V Palit, P Winyard, H Bedford. UCL Great Ormond Street Institute of Child Health, London, UK

\subsection{6/archdischild-2018-rcpch.114}

Background Rapid post-natal growth is associated with increased risk of adult cardiovascular and metabolic disease. In preterm infants, rapid post-natal growth is also associated with improved long-term cognition. Small-for-gestational-age (SGA) term infants have increased risk of adverse long-term cognitive outcomes compared to appropriate-for-gestational-age (AGA) term infants; they are also prone to rapid post-natal 'catch-up' growth. It is therefore important to understand whether rapid post-natal growth in this population confers any long-term cognitive advantage, and balance this with recognised metabolic risks.

Aims To investigate associations between post-natal growth in term-SGA infants and cognitive outcomes in adolescence.

Methods 60 term-SGA infants were followed-up from birth to 16 years. Weight, head circumference (HC) and length were measured at enrolment, 6, 12 and 26 weeks, 9 and 18 months and 16 years. Measurements were converted to standard deviation scores (SDS) and changes in SDS between time points calculated. Cognitive outcomes were measured at 16 years to assess global intelligence (IQ); literacy and academic attainment (Wechsler Individual Attainment Test-reading and maths subtests); and executive functioning (Stroop Colour-
Word test). Univariate and multivariate analyses explored associations between growth and cognitive outcomes.

Results In univariate analysis HC growth from 0-6 weeks was significantly associated with increased Full Scale and Verbal IQ (FSIQ and VIQ). A 1-SDS increase in HC growth over this period was associated with a 7.10-point increase in FSIQ (95\% CI: 1.15 to $13.04, \mathrm{p}=0.02$ ) and a 10.82 -point increase in VIQ (95\% CI: 3.31 to 18.34), $\mathrm{p}=0.006$. However, after adjustment for confounders in multivariate models, HC growth no longer significantly predicted cognitive outcome, whilst maternal education was a significant predictor of IQ, reading and maths scores.

Conclusions A positive association between HC growth from birth to 6 weeks and later IQ was seen. However, this was not significant after adjustment for confounding factors, possibly due to under-powering. Maternal education was a significant predictor of cognitive outcomes at 16 years. Given the recognised risks associated with rapid post-natal growth, this study does not support promoting rapid growth in term-SGA infants.

\section{G119(P) IN-SITU PAEDIATRIC SIMULATION FOR MEDICAL STUDENTS}

K Douch, C Branthwaite, G Thian, B Morrissey. Paediatrics, Royal London Hospital, London, UK

\subsection{6/archdischild-2018-rcpch.115}

Aims Medical students feel unprepared to assess and manage unwell patients, particularly children. We designed an innovative simulation programme, consisting of four interactive paediatric cases, to help medical students learn to recognise serious illness in children and institute appropriate management, and to support them developing skills in prescribing for children, clinical reasoning, team-working and handover. We evaluated the impact of this programme on medical student's confidence in recognising and managing sick children.

Methods We trialled the simulation programme on fourth year medical students undertaking their paediatric placement at a London teaching hospital. Scenarios were run in-situ in realtime in a cubicle on the paediatric short-stay unit, and were facilitated by paediatric trainees and consultants. Students took part in four scenarios, over two sessions. All students had previously attended a workshop on recognition and management of the sick child during their child health teaching week.

Students were asked to complete an anonymous survey rating their confidence in 13 areas before and after taking part in the programme. They rated their confidence from one (not confident) to five (highly confident.) They were also asked their views on the programme and learning points.

Results Prior to completing the programme, students were least confident in prescribing emergency medications for children and recognising and managing a child with seizures and shock. Self-assessed confidence in recognising a sick child increased from 2.1 to 3.3 after completing the programme. In all areas, students reported a statistically significant increase in their confidence, particularly in working as a multi-disciplinary team to manage a sick child. Students left comments that the sessions were hugely helpful especially in learning a systematic approach to assess and manage a sick child, prescribing for children, communication and teamwork. Paediatric trainees who facilitated the programme gained skills in leadership, management and facilitation. 